\title{
TEORÍA Y ESTUDIO DE LA CORRESPONDENCIA COMERCIAL EN LENGUA ALEMANA
}

\author{
Alfonso Corbacho Sánchez \\ Universidad Politécnica de Valencia
}

\begin{abstract}
The purpose of this study is to highlight the importance of commercial correspondence in German. It is interesting to note that there are not many linguistic-based studies describing the features of this particular type of writing. We contribute here an overview of business correspondence including specific vocabulary and its linguistic structures. The primary focus of our work is on lexical, morphological and syntactic aspects.
\end{abstract}

\section{INTRODUCCIÓN}

Actualmente, el mundo de los negocios y, más concretamente, la empresa y su correspondiente actividad económica representan el motor de empuje de cualquier sociedad avanzada. Claro está, que en los sistemas económicos la comunicación escrita, por antonomasia, es la carta comercial, dado que "ein Großteil der Geschäfte über diese Kommunikationsformen abgewickelt wird” (Hahn, 1993: 95). Por consiguiente, dominar la redacción de la correspondencia comercial en el ámbito empresarial no sólo se antoja fundamental, sino también imprescindible, ya sea a través del correo ordinario, fax, telegrama o de herramientas informáticas como es el correo electrónico.

En cuanto a la correspondencia comercial en lengua alemana, ésta sirve de enlace entre Alemania, país que copa el primer puesto en la economía de Europa, y el resto de las naciones integrantes de la Unión Europea y demás países del mundo.

Es objetivo de esta exposición teorizar sobre las estructuras de la carta comercial que, en definitiva, no es una técnica que destaque por su alto grado de dificultad habida cuenta de la "Bausteinverarbeitung" . La correspondencia comercial se puede definir como aquélla que posibilita la comunicación en el mundo de los negocios por medio de la escritura y en este contexto Sachs perfila (1994: 10) que la "Bausteinverarbeitung" consiste en:

"die Speicherung von Briefteilen in einem Textsystem, die dann nach Bedarf in einer bestimmten Reihenfoge abgerufen und zu einem Brief kombiniert werden können”.

\footnotetext{
${ }^{1}$ Dicho de otro modo, de las cartas obtenemos los denominados "Textbausteine", que son las estructuras fijas que con mayor frecuencia se repiten y que pueden ser sustituidas por otras similares.
} 
Por tanto, en el estudio de esta disciplina lingüística lo fundamental reside en dominar el mayor número de "Textbausteine" o "Briefbausteine" con la finalidad de poder redactar varios modelos de cartas diferentes.

No obstante, no podemos olvidar que la redacción comercial está situada en el marco de los lenguajes especializados, concretamente, en el lenguaje económico. Y como nuestro estudio es confeccionado desde la perspectiva de la lengua alemana, el siguiente punto de nuestro tema tratará de definir el lenguaje económico alemán.

\section{El LENGUAJE ECONÓMICO ALEMÁN}

La economía desempeña un papel primordal en los asuntos cotidianos del hombre y no sólo en las actividades económicas de nuestra era, sino a lo largo de la historia de las civilizaciones. Ciertamente, que la naturaleza de los problemas, que arrancan de la economía, ha cambiado por el paso del tiempo, pero algunas lacras que padece nuestra sociedad como la pobreza, alcanzan tintes universales e intemporales. Sin embargo, también derivan aspectos positivos de esta área de la actividad humana como es el progreso.

Pues bien, como en cualquier otro campo del conocimiento, el progreso y los lenguajes especializados experimentan una evolución paralela. La actividad en el mundo de los negocios origina un caudal copioso de términos específicos imprescindibles a la hora de denominar conceptos novedosos que posibilitan la comunicación en el ámbito profesional de la economía.

Para aclarar este punto con mayor precisión, y siempre en el marco de la lengua alemana, consideraremos la cuestión sin detenernos a teorizar sobre el concepto de los lenguajes especializados. Diversas definiciones se han dado por los estudiosos al concepto genérico de lenguaje económico, aunque tal vez sea la definición de Buhlmann y Fearns (1987:306) una de las más acertadas:

"Wirtschaftssprache, das ist die Gesamtheit aller Fachsprachen, d. h. aller sprachlichen Mittel, die in einem fachlich begrenzten Kommunikationsbereich, nähmlich dem der Wirtschaft, verwendet werden, um die Verständigung der in diesem Bereich tätigen Menschen zu gewährleisten".

Según esto el lenguaje económico es el conjunto de lenguajes especializados referidos al ámbito de la economía que hacen posible la comunicación y el entendimiento entre los especialistas. Así, Buhlmann (1989: 86) en su intento de definir el lenguaje económico desde la óptica del idioma alemán, vuelve a expresarse en los mismos términos cuando escribe que el lenguaje especializado de la economía:

"... die Summe der in der Wirtschaft bzw. in der wirtschaftsbedingten Kommunikation benutzten Fachsprachen ist".

Como toda definición sintética puede quedar a veces falta de matices importantes. Con ello nos referimos a que Buhlmann restringe y delimita en exceso el área de actuación del lenguaje económico al ámbito de los expertos. Recuérdese que la economía forma parte de la actividad cotidiana y no todos los contextos hacen gala de un léxico específico. En 
consecuencia, una parcela determinada del lenguaje que es objeto de estudio sí está al alcance de cualquier lego en la materia.

El estudioso Hahn (1993: 92) en su definición de alemán económico expresa magistralmente la idea que compartimos más arriba, englobando todos los medios lingüísticos que se utilizan en el campo de la economía, tanto los aspectos científicos y teóricos del lenguaje económico alemán como aquellos otros contenidos pertenecientes al área de la economía que pueda presentar la lengua común. En sus palabras:

"Wir verwenden den allgemeinen Begriff "Wirtschaftsdeutsch" und meinen damit sowohl die wirtschaftsbezogene Fachsprache im wissenschaftlich-theoretischen Bereich als auch die Berufssprachen in der Wirtschaft und die fachbezogene Umgangssprache".

En la misma tesitura, nos topamos con la definición dada por van Megen (1989: 232) que reitera muy bien esta visión del campo de estudio del lenguaje económico alemán, refiriéndose, sobre todo, a la actividad comercial. Y escribe:

"Die Wirtschaftssprache umfaßt im weitesten Sinne die gesamten Fachsprachen, welche sich auf die verschiedenen Fachgebiete des Handels und der Industrie beziehen. Dieser Fachsprache bedienen sich Unternehmer, Verhandlungspartner, Mitarbeiter, im Kontakt mit Fachkollegen und mit anderen, etwa Kunden und Besuchern, um zu informieren, zu beschreiben, zu verhandeln, um Produktion, Absatz oder Dienstleistung zu verbessern".

En conclusión, podemos constatar que "Wirtschaftsdeutsch ein Konglomerat von wirtschaftsbezogenen Fachsprachen darstellt” (Schaarschuh, 1991:144). En otras palabras, el lenguaje económico alemán está integrado en el amplio grupo de los lenguajes especializados y, a su vez, abarca diversos lenguajes especializados al alcance de un amplio colectivo de personas, tanto especialistas como profanos, en el heterogéneo sector de la economía. Por consiguiente, nos atrevemos a confirmar la existencia del alemán económico como lenguaje especializado por la acumulación de rasgos definitorios propios y precisos que pueden sustentar y defender este principio.

\section{ESTRUCTURA DE LA CARTA COMERCIAL ALEMANA}

A continuación procedemos a exponer el orden estructural de la carta comercial más aceptado, aunque, naturalmente, llegar a un modelo generalizado y estándar de la distribución de las distintas partes que la conforman resulta un tanto complicado, pues todo depende de las preferencias de los usuarios del presente código lingüístico. Así pues, la carta comercial queda constituida por las siguientes partes:

a) DER BRIEFKOPF (el membrete)

Las empresas suelen utilizar el papel con membrete, situado en la parte superior de la carta, cuya finalidad es dar a conocer al remitente. Al margen de cualquier diseño gráfico de la marca comercial, logotipo o anagrama, incluye los siguientes elementos:

"der Firmenname" (la razón social) y “die Gesellschaftsform” (la forma de sociedad)

"der Geschäftszweig" (la actividad o el ramo) 
"die Anschrift / die Adresse" (la dirección). Conviene apuntar que "die Postleitzahl" (el código postal) va precedido de la sigla " $D$ " (Deutschland) seguido de un guión, cuando se mantiene correspondencia comercial con países extranjeros.

b) DIE ANSCHRIFT / DIE ADRESSE DES EMPFÄNGERS (la dirección del destinatario)

El nombre y la dirección del destinatario están ubicados a la izquierda debajo del membrete. Cuando la carta va dirigida a una persona determinada, se escribe "Herrn" (Sr.) o "Frau" (Sra.), que podrán ir acompañados, en la misma línea, de la profesión o la función que desempeña el destinatario en la empresa. Asimismo, los títulos académicos, situados en la línea siguiente en abreviatura (Dr.- "Doktor") son parte integrante del nombre, por lo que no deben omitirse, ya que en Alemania gozan de gran prestigio.

Debajo del nombre se escribirá la calle y el número, que sólo van separados por un espacio sin que deba figurar una coma. En la línea siguiente el código postal y la población como ya hemos mencionado más arriba.

En el supuesto de que el destinatario indique en su dirección el "Postfach" (apartado de correos), se suprimirá la calle y el número.

Por otra parte, si la carta va dirigida a empresas se podrá escribir "Firma" (empresa) o bien indicar el nombre de la empresa en mayúsculas. Igualmente podrá incluirse el departamento, sección u oficina correspondientes (Abteilung) a los que se dirija la carta. Sin embargo, si la misiva va destinada a una persona determinada, debe aparecer "zu Händen”, abreviado “z. Hd.” (a la atención de).

Demás indicaciones del servicio postal que se pueden leer en un sobre son: "Bitte nachsenden" (reexpedir al destinatario), "Drucksache" (expreso), "Eilboten", "eilt", "dringend" (correo urgente), "Eilsendung", "Eilbrief" (expreso), "Einschreiben" (certificado), "Einschreiben gegen Rückschein" (certificado con resguardo), "Luftpost" (por avión), "Nachnahme" (contra reembolso), "vertraulich" (confidencial), "streng vertraulich" (estrictamente confidencial).

c) DIE BEZUGSZEICHEN (las referencias internas)

Las referencias internas facilitan la identificación de la carta. Aparecen tanto la referencia del destinatario (Ihr Zeichen), que suelen ser las iniciales en mayúsculas correspondientes al nombre y apellido de la persona a la que va dirigida la correspondencia, como la del remitente (Unser Zeichen), que, por lo general, son las iniciales de la persona responsable que ha dictado la carta seguidas de una barra y las de la persona encargada de haber escrito la comunicación.

d) DAS DATUM (la fecha)

En lo que respecta a la fecha, ésta debe figurar en la misma línea de las referencias internas, normalmente va precedida del nombre de la localidad seguida de una coma. El día se escribirá en números; el mes en números o en letras, teniendo en cuenta que el punto que

\footnotetext{
2 "Fräulein" (señorita) está cada vez más en desuso, por lo que se utiliza la indicación "Frau".
} 
sigue al día y al mes nos indica que se trata de un ordinal. Y por último, el año que aparecerá escrito en números. Citamos como ejemplos ${ }^{3}$ :

Düsseldorf, den 15. Dezember 2001

Düsseldorf, 15. Dezember 2001

Düsseldorf, 15. 12. 2001

e) DER BETREFF (la referencia / el asunto)

La referencia o el asunto es una indicación breve, una o dos palabras, que nos adelanta el contenido de la carta y facilita la distribución de correo dentro de una empresa, a su vez, asegura la rápida identificación del mensaje sin la necesidad de tener que realizar una lectura de los primeros párrafos.

f) DIE ANREDE (el tratamiento)

Una de las características de los escritos comerciales es su estilo formulario. Así ocurre con el tratamiento, que depende en muchas ocasiones de la relación entre el remitente y el destinatario, cuando este último es una persona conocida, se utiliza la siguiente fórmula acompañada del nombre en cuestión:

- "Sehr geehrter Herr" (Estimado señor)

- "Sehr geehrte Frau" (Estimada señora)

Sin embargo, en los casos que se desconoce al interlocutor, se recurre a la fórmula estándar "Sehr geehrte Damen und Herren" (Estimados Sras. y Sres.) para las cartas a empresas comerciales, sociedades y autoridades. Todo ello seguido de una coma y con minúscula. Así lo afirma Reichel (1995: 50) cuando escribe:

“... benutzen Sie die allgemeine Anrede "Sehr geehrte Damen und Herren". Heute ist es üblich, hinter die Anrede ein Komma zu setzen und (in der übernächsten Zeile) klein weiterzuschreiben".

Además, cabe otra posibilidad menos formal y es cuando la correspondencia comercial se lleva a cabo con destinatarios con los que existe una óptima relación comercial. En este caso, se utilizarán las expresiones:

"Lieber Herr" (Querido señor)

"Liebe Frau" (Querida señora)

g) DER BRIEFTEXT (el cuerpo de la carta)

Obviamente es el centro neurálgico de toda comunicación escrita. El texto, estructurado en párrafos bien delimitados que corresponden a las diversas unidades de sentido (Karlson / Karlson, 1994: 9), debe guardar un espacio en blanco del saludo o tratamiento. Del mismo modo, se procederá con los diferentes párrafos que conforman la carta comercial. Además,

\footnotetext{
${ }^{3}$ Nos indica Sachs (1994: 12) que la norma ISO aconseja para la fecha la secuencia siguiente: año-mes-día, por ejemplo: 2001-12-15.
} 
cabe apuntar que la redacción comercial alemana carece de la típica sangría en la primera línea a lo largo de todo el texto.

El cuerpo de la carta es la parte fundamental de la correspondencia comercial, en la que es costumbre destinar un párrafo conciso y distinto a cada uno de los puntos tratados. Sus frases deben cumplir, sobre todo, los objetivos de la claridad expositiva y la brevedad de acuerdo a un orden lógico y coherente, que paulatinamente desarrolle los contenidos más relevantes, eludiendo circunloquios, reiteraciones innecesarias, imprecisiones y todo tipo de recursos retóricos.

Otro rasgo que tiene gran importancia en la redacción de este tipo de cartas es el afán de precisión. Se buscará la precisión y lo concreto en el motivo de la misiva, de manera que evitaremos todo detalle secundario que se aparte del contenido esencial y conduzca a significados ambiguos que dificulten la comprensión. Por ello, es recomendable abordar directamente el propósito de la carta y evitar la inclusión de diversos asuntos que puedan dificultar su entendimiento.

h) DIE SCHLUSSFORMEL (la fórmula de despedida)

Las fórmulas de despedida, que acostumbran ir enlazadas con alguna frase o expresión de cierre ("In Erwartung Ihrer Antwort verbleiben wir" - Quedamos a la espera de sus noticias), se caracterizan por su extrema brevedad y su cordialidad. Destacamos entre muchas fórmulas aceptadas "mit freundlichen Grüßen" ( saludos cordiales) por ser la más empleada, recordando que los saludos finales se escriben en el margen izquierdo. Otra fórmula más respetuosa y solemne es "hochachtungsvoll" (atentamente) que se usa "cuando el destinatario es desconocido o cuando existe una relación jerárquica implícita" (Boelcke, 1998: 15). Y, por último, aún más rígida y severa resulta "mit vorzüglicher Hochachtung" (muy atentamente / con el mayor aprecio) para correspondencias muy formales.

\section{i) DIE UNTERSCHRIFT (la firma)}

A continuación de las fórmulas de despedida nos topamos con la firma del remitente, justo encima del nombre y la categoría profesional mecanografiados. En el caso de que existan personas delegadas la firma puede ir precedida de "ppa o pp" (per procura / por poder), "i. V." (in Vollmacht / por autorización) e "i. A." (im Auftrag / por orden), según corresponda.

j) DIE ANLAGEN (el anexo / los documentos adjuntos)

Cualquier documento adjunto a la carta recibe el nombre de anexo. Cuando se adjuntan documentos como facturas, certificados, recibos, etc., figuran al pie de la carta, normalmente a la izquierda, con la palabra "Anlage(n)" subrayada.

k) EL PIE DE LA CARTA (Der Brieffu $\beta$ )

Al pie de las cartas comerciales pueden figurar datos como números de teléfono, número de fax y cuentas bancarias entre otros. 


\section{RASGOS LINGÜÍSTICOS DE LA CORRESPONDENCIA COMERCIAL ALEMANA}

El lenguaje de la correspondencia comercial es exclusivamente escrito, que, a pesar de nutrirse de la lengua común, manifiesta una serie de particularidades léxico-semánticas y morfosintácticas propias. A continuación señalaremos con carácter descriptivo cuáles son los principales rasgos lingüísticos de la correspondencia comercial alemana desde los dos niveles mencionados.

\subsection{Nivel léxico-semántico}

a) El lenguaje de la correspondencia comercial, como los demás lenguajes especializados, en su intento de favorecer la precisión y la claridad imponen una terminología unívoca, consistente en que a un término le corresponde un único significado. Así, se genera el uso de términos monosémicos y unívocos, es decir, tecnicismos, que son toda la terminología propia de una determinada especialidad. Tal vez, en el lenguaje de la correspondencia comercial no se da un abultado número de tecnicismos, en sentido estricto, como sucede en otros campos del saber, pues la mayoría de éstos son vocablos de la lengua común de uso generalizado, que en un entorno comercial sí pueden adquirir un significado particular con importantes diferencias de matiz. Sin embargo, los tecnicismos también pueden registrar un léxico específico que no proceda del lenguaje común. Thomas Roelcke se sitúa más allá de estos presupuestos y simplifica la definición de tecnicismo, posicionándolo dentro de las dimensiones del texto, sin olvidar el componente comunicativo. De este modo, nos puntualiza que:

"Ein Fachwort ist hiernach die kleinste bedeutungstragende und zugleich frei verwendbare sprachliche Einheit eines fachlichen Sprachsystems, die innerhalb der Kommunikation eines bestimmten menschlichen Tätigkeitsbereichs im Rahmen geäußerter Texte gebraucht wird". (Roelcke, 1999: 51)

No obstante, al margen de la posibilidad que conlleva el empleo más o menos específico de un tecnicismo, la línea trazada en la correspondencia comercial es aquélla que se inclina hacia la claridad y la sencillez.

b) También es característica el empleo de anglicismos ${ }^{4}$, es decir, vocablos procedente de la lengua inglesa, que en la actualidad penetran en el léxico de todas las lenguas. Asimismo, merece especial atención la invasión de términos provenientes de la informática, disciplina que se ha convertido en un auténtico manantial de terminología novedosa, filtrándose sin contemplaciones en todas las lenguas del mundo, sobre todo, por el peso cada día más específico del comercio electrónico. Por otra parte, también distinguiremos en este apartado entre préstamo, palabra que se toma de otra lengua sin proceder a su traducción con posibles adaptaciones morfológicas y fonéticas, y calco, palabra que se recoge y traduce literalmente de otra lengua. Incluso cabe la posibilidad de encontrarnos con ambos fenómenos lingüísticos en la misma palabras .

\footnotetext{
${ }^{4}$ Véase el estudio detallado de anglicismos "Wieder: Die Engländerei in der deutschen Sprache” de Carstensen.

${ }^{5}$ Consúltese el interesantísimo artículo "Préstamo y calco en español y alemán. Su interés lingúístico y su tratamiento en la traducción" de García Yebra, donde describe con sumo detalle las opciones combinatorias existentes entre préstamo y calco.
} 
- "Marketing”, "Holding”, “E-Mail”, "Leasing”, "Export”, etc.

c) Las abreviaturas son empleadas con frecuencia por obvias razones de economía lingüística:

- “Abs." (Absender), "Abt." (Abteilung), "Fa." (Firma), "i.A.” (Reyero / Rohr, 2001: 110)

d) El lenguaje de la correspondencia comercial resulta en muchas ocasiones un lenguaje formulístico cuajado de giros estereotipados. Estas expresiones fijadas o fórmulas fraseológicas pueden quedar distribuidas a lo largo de todo el texto, pero, sobre todo, en el comienzo y final de la carta comercial.

- "In Beantwortung Ihres Schreibens vom ..." (Schmitz / Scheiner, 1996: 19)

- "Für weitere Auskünfte stehen wir Ihnen zur Verfügung" (Karlson/Karlson, 1994: 50)

e) Las siglas en nuestro tiempo parecen poseer una entidad propia, pues resulta casi insólito el hecho de que no podamos apreciar uno de estos elementos en la redacción comercial. De esta manera, existe una clara tendencia a crear términos por acronimia. Entendemos por acrónimo aquellas palabras formadas a partir de las siglas de una palabra. Este recurso lingüístico es utilizado cuando se alude a instituciones y organismos.

"AG" (Aktiengesellschaft), "GmbH" (Gesellschaft mit beschränkter Haftung) (Reyero / Rohr, 2001: 110)

"BIZ" (Bank für internationalen Zahlungsausgleich), "DAAD" (Deutscher Akademischer Austauschdienst) (Boelcke, 1998: 234)

f) Presencia de términos construidos por prefijación y sufijación. Destacamos en particular la terminación en -er que hace expresa indicación a personas:

- “Absender", "Empfänger”, etc.(Reyero / Rohr, 2001: 33)

g) Aparición de términos compuestos. La lengua alemana es proclive a la formación de palabras compuestas frente a otras lenguas.

- "Zusammenarbeit”, “Zahlungsverpflichtungen", "Vertragsänderung”, "Stellenangebot", "Preisermäßigung”, etc.

\subsection{Nivel morfosintáctico}

a) Por lo que respecta a tiempos y modos, sobresalen los siguientes:

- el presente de indicativo:

- "Hiermit bitte ich um Zusendung von ..." (Schmitz / Scheiner, 1996: 34)

- el imperativo:

. "(bitte) übersenden Sie mir (auch) ...", "schicken Sie uns bitte Ihre Preisliste für ...” (Schmitz / Scheiner, 1996: 25)

- el Konjunktiv II: 
- "Ich wäre Ihnen sehr dankbar, wenn Sie mir bald Bescheid geben könnten" (Schmitz / Scheiner, 1996: 48)

- Igualmente por medio de la perifrasis "würde + infinitivo":

- "Wir würden uns sehr freuen" (Reyero / Rohr, 2001: 31)

- el futuro:

- "Die Mehrkosten werden wir Ihnen dann in Rechnung stellen" (Hering / Matussek, 1996: 9

b) En el ámbito de la correspondencia comercial alemana se utiliza el tratamiento de cortesía de la $2^{\circ}$ persona, tanto del singular como del plural, "Sie" con la finalidad de mantener la cortesía entre los corresponsales.

- "Wie Sie aus unserem Schreiben erfahren haben, ..." (Karlson / Karlson, 1994: 39)

c) Propensión al uso de construcciones nominales ${ }^{7}$. Esta tendencia se caracteriza por el uso abundante de sustantivos frente a otras categorías gramaticales. Así lo advierte el lingüísta Fluck (1985: 48) cuando escribe que: "Die wichtigste Wortart unter den spezialsprachlichen lexikalischen Einheiten bilden die Substantive". A este respecto, citamos la construcción formada por un verbo seguido de un sustantivo en sustitución de nociones verbales:

"in Verbindung setzen", "ins Defizit geraten", "ein Konto schließen"

d) El uso de la adjetivación se restringe a cuando es necesario, siendo su función descriptiva, y contribuyendo a la precisión del escrito comercial.

- "aktuelle Preisliste", "exzellenter Geschäftspartner", "finanzielle Schwerigkeiten"

e) Empleo del Partizip I (participio de presente ${ }^{8}$ ) como adjetivo atributivo:

- "einen entsprechenden Nachlass" (Schmitz / Scheiner, 1996: 41)

- y como adverbio de modo:

- "Wir bestätigen hiermit dankend Ihren Brief vom ..." (Schmitz / Scheiner, 1996: 69)

f) Es un rasgo muy característico del estilo comercial alemán, y de la lengua alemana en general, el empleo de la pasiva, asegurando la objetividad y ocasionando la consiguiente despersonalización de la oración al evadir la responsabilidad directa de quien realiza la acción.

\footnotetext{
${ }^{6}$ Advierten Corcoll / Corcoll (1994: 215) que desde un punto semántico, se debe considerar el pronombre "Sie" como $2^{\circ}$ persona.

${ }^{7}$ Un estudio completo del estilo nominal lo encontraremos en la obra de Punkki - Roscher, M. (1995), Nominalstil in populärwissenschaftlichen Texten: zur Syntax und Semantik der komplexen Nominalphrasen, Peter Lang, Frankfurt am Main.

${ }^{8}$ Véase Corcoll / Corcoll (1994: 135 y sigs.).
} 
- "Er wurde bei unserer Bank zahlbar gestellt" (Hering / Matussek, 1996: 87)

g) Uso de construcciones con verbos seguidos de complemento preposicional:

- "bitten um (+Akk.), sich informieren über (+Akk.), sich eindecken mit (+Dat.)" (Reyero / Rohr, 2001: 24)

h) La modalidad de la oración en la correspondencia comercial es, en exclusividad, afirmativa-enunciativa, adecuada para toda exposición. En las oraciones impera la precisión y la claridad, predominando las compuestas frente a la oración simple. En el campo de la oración compuesta señalamos las proposiciones más frecuentes:

- la oraciones de relativo:

- "Die Firmen, die ich vertreten habe, werden über mich Auskunft geben" (Boelcke, 1998: 178).

- las oraciones de infinitivo con $z u$ :

- "Wir bitten Sie uns den Termin umgehend mitzuteilen" (Reyero / Rohr, 2001: 33)

- oraciones subordinadas con la conjución "dass":

- "Wir garantieren Ihnen, dass wir die Rechnung zum neuen Termin begleichen werden" (Hering / Matussek, 1996: 136)

- las oraciones interrogativas indirectas:

- "Bitte teilen Sie uns mit, ob Sie mit diesem Vorschlag einverstanden sind" (Hering / Matussek, 1996: 104)

- "Wir möchten wissen, wann die Lieferung erfolgt" (Reyero / Rohr, 2001: 32)

i) Proliferan los verbos modales:

- "Zu unserem großen Bedauern müssen wir diese Reservierung annullieren" (Barberis / Bruno, 1989: 74)

- "Wir möchten so schnell wie möglich mit Ihnen in Geschäftsverbingung treten" (Boelcke, 1998: 38)

j) Sustantivación de infinitivos en -ung.

- "Anschaffung”, “Aufforderung”, "Barzahlung", "Einstellung”, "Fertigung”, "Kündigung”, "Überweisung", etc.

k) Aparición de medios extralingüísticos9. La carta comercial puede contener algún elemento icónico, concretamente, en lo que respecta al membrete de la empresa.

\footnotetext{
${ }^{9}$ Véase a Fluck (1985: 210) y Roelcke (1999: 92).
} 


\section{Tipos de Cartas Comercialles alemanas}

Una vez analizados los rasgos más frecuentes del estilo comercial en el idioma alemán, sólo nos queda incorporar al presente trabajo los textos comerciales en los que se exteriorizan los rasgos lingüísticos señalados.

Las cartas comerciales nos ofrecen información sobre una larga lista de operaciones mercantiles que se reflejan en los diferentes tipos de cartas comerciales. En consecuencia, es de buena lógica encuadrar esta tipología textual dentro del campo de los textos especializados, lo que nos encamina a detallar los diversos modelos de la correspondencia comercial desde el punto de vista del texto, es decir, de la lingüística textual.

Si interpretamos el texto como la unidad supraoracional en el acto comunicativo, ahora nos posicionaremos en un ámbito de actividad más específico que posibilita la comunicación entre especialistas. En esta línea, Roelcke (1999: 42) nos aclara el concepto de la siguiente manera:

"Unter Fachtexten sind dabei komplexe und zugleich kohärente sprachliche Äußerungen (oberhalb der Satzgrenze) im Rahmen der Kommunikation innerhalb eines bestimmten menschlichen Tätigkeitsbereich zu verstehen".

Por tanto, podemos considerar toda la comunicacion escrita en un entorno comercial como textos especializados. A este respecto, retomando el concepto de alemán económico, transcribimos la cita de Frenser (1991: 127), en la que se apoya en textos para configurar su definición del lenguaje económico alemán. Y nos dice que:

"Wirtschaftsdeutsch setzt sich zusammen aus Texten der Fachsprache, der komplexen Berufssprachen und aus Texten, die Wirtschaft zum Thema haben".

Por último, hacemos constatar que una exposición exhaustiva y una clasificación rigurosa de los diferentes modelos de la correspondencia comercial nos ocuparía un espacio de dimensiones considerables por los numerosos subapartados que puede registrar cada carta comercial. Por ello, nos limitamos a citar algunos textos, que suelen caracterizarse por la finalidad que persiguen, entre los más destacados: "Angebote" (Ofertas), "Stellenangebote" (Ofertas de trabajo), "Anfragen" (Solicitudes), "Bewerbung" (Solicitud de empleo), "Lebenslauf" (Currículum vitae), "Liefererung" (Suministro), “Zahlung” (Pago), "Bestellung" (Pedidos), "Auftragsbestätigung" (Confirmación de Pedidos), "Kostenvoranschläge" (Presupuestos), "Rechnungausstellung" (Facturación), "Mahnungen" (Reclamaciones de Pago), "Kreditauskunftsersuchen" (Solicitud de Información sobre Solvencia), "Beschwerden - Reklamationen" (Reclamaciones), "Versicherung und Garantie" (Seguros y Garantías), "Rundschreiben" (Circulares), "Reservierung" (Reservas).

\section{CONCLUSIÓN}

Para cerrar la presente exposición nos gustaría comentar que nuestra pretensión ha consistido en trazar las líneas principales de la correspondencia comercial en lengua alemana, intentando con ello no solamente resaltar la clara tendencia ascendente de esta disciplina sino también incidir en su investigación lingüística para la consecución de un 
conocimiento más profundo del lenguaje epistolar. Del mismo modo, compartimos la idea de que uno de los principales cometidos de un filólogo también consiste en saber responder de modo profesional a todas las exigencias que le puede deparar una situación propia del mercado laboral o del mundo de los negocios.

Llegados a este punto son muchos los motivos que podríamos indicar a favor de nuestro objeto de estudio. En la actualidad, lo que justifica con creces la constante expansión de la correspondencia comercial es su inclusión en las nuevas tecnologías de la información y de la comunicación bajo el nombre de correo electrónico o el ya internacionalizado e-mail. Salvando los obstáculos de ciertas características propias de los mensajes electrónicos como pueden ser la utilización de símbolos y de normas paralingüísticas -tema que por sí mismo ya merece un estudio por separado-, el contenido de los textos electrónicos no presenta grandes diferencias con respecto a aquellos de corte más tradicional. Con ello, se nos continúa abriendo aquí otra parcela más que nos induce a dominar las reglas generales de las epístolas comerciales.

\section{BIBLIOGRAFÍA}

BARBERIS, P. / E. BRUNO (1989), Deutsch im Hotel. Kommunikatives Lehrwerk für Deutschlerner im Fach Hotelgewerbe. Korrespondenz führen (Teil 2), Hueber, Ismaning.

BOELCKE, J. et al. (1998), Correspondencia comercial en alemán, Larousse, Barcelona.

BUHLMANN, R. / A. FEARNS (1987), Handbuch des Fachsprachenunterrichts. Unter besonderer Berücksichtigung naturwissenschaftlich-technischer Fachsprachen, Langenscheidt, München.

BUHLMANN, R. (1989), “Fachsprache Wirtschaft-gibt es die?”. En: Jahrbuch Deutsch als Fremdsprache, 15, págs.82-108.

BUHLMANN, R. (1994), "Lenguaje económico. Características didácticamente relevantes y consecuencias para la formación y capacitación de profesores". En: Alejo, R./M. McGinity/S. Gómez (eds.), Lenguas para Fines Específicos:temas fundamentales, Universidad de Extremadura, págs. 69-80.

CARSTENSEN, B. (1984), "Wieder: Die Engländerei in der deutschen Sprache". En: Die deutsche Sprache der Gegenwart, Joachim Jungius-Gesellschaft, Hamburg, págs. 4357.

CORCOLL, B. / R. CORCOLL (1994), Programm. Alemán para hispanohablantes (Tomo I), Herder, Barcelona.

DESINGER, B. et al. (eds.) (1999), Basiswissen Wirtschaftsdeutsch. Stoffgebiete und Fachwortschatz, Iudicium, München.

FLUCK, H.-R. (1985), Fachsprachen, Francke Verlag, München.

FRENSER, U. (1991), "Landeskunde in Wirtschaftsdeutschlehrwerken". En: Informationen Deutsch als Fremdsprache, 2, págs. 125-135. 
GARCÍA YEBRA, V. (1987), "Préstamo y Calco en Español y Alemán. Su interés lingüístico y su tratamiento en la traducción”. En: Problemas de la traducción (Mesa redonda - Nov. 1983), Fundación Alfonso X “El Sabio”, Madrid, págs. 75-89.

HAHN, M. (1993), "Deutsch als Wirtschaftssprache”. En: Deutsch als Fremdsprache, 2, págs.92-95.

HERING, A. / M. MATUSSEK (1996), Geschäftskommunikation. Schreiben und Telefonieren, Hueber, Ismaning.

KARLSON, L. / L. KARLSON (1994), Escribir cartas - ALEMÁN, Difusión, Barcelona.

MEGEN, J. v. (1989), "Wirtschaftsdeutsch". En: Jahrbuch Deutsch als Fremdsprache, 15, págs. 225-236.

OHNACKER, K. (1991), "Die Syntax der Fachsprache Wirtschaft”. En: Zielsprache Deutsch, 4, págs. 221-230.

PIIRAINEN, I. T. (1982), "Die Sprache der Wirtschaftspresse". En: Muttersprache, 92, págs. 27-37.

PUNKKI - ROSCHER, M. (1995), Nominalstil in populärwissenschaftlichen Texten: zur Syntax und Semantik der komplexen Nominalphrasen, Peter Lang, Frankfurt am Main.

REICHEL, W. (1995), Berufsstart für Hochschulabsolventen. Erfolgsstrategien für Bewerbung und Vorstellung, Falken Verlag, Niedernhausen.

REYERO HERNÁNDEZ, C. / K. ROHR SCHRADE (2001), Geschäftsbriefe? Kein Problem! Elementare Geschäftsbriefe für Deutsch als Fremdsprache, Dirección General de Universidades e Investigación, Santa Cruz de Tenerife.

ROELCKE, T. (1999), Fachsprachen, Erich Schmidt Verlag, Berlin.

SACHS, R. (1994), Deutsche Handelskorrespondenz. Der Briefwechsel in Export und Import, Hueber, Ismaning.

SCHAARSCHUH, F.-J. (1991), "Wirtschaftsdeutsch-deutsche Sprache der Wirtschaft?". En: Deutsch als Fremdsprache, 3, págs.140-145.

SCHMITZ, W. / D. SCHEINER (1996), Ihr Schreiben vom ... Geschäftliche und private Briefe im Baukastensystem, Verlag für Deutsch, Ismaning / München.

V.V. A.A. (1996), La nueva correspondencia comercial español-alemán, De Vecchi, Barcelona. 
